\title{
25 Research Square \\ The Association Between Serum Uric Acid and the Cardiometabolic Phenotype Among Healthcare Workers in Azar Cohort Study
}

\section{Mohammad hossein Somi}

liver and Gastrointestinal Diseases Research center, Tabriz University of Medical Sciences

\section{Negin Frounchi}

Student Research Committee, Tabriz University of Medical Sciences

\section{Alireza Ostadrahimi}

Nutrition Research Center, Tabriz University of Medical Scinces

\section{Seyed Sina Zakavi}

Student Research Committee, Tabrzi University of Medical Sciences

\section{Neda Gilani}

Department of Statistics and Epidemiology, Facualty of Health , Tabriz University of Medical Sciences Elnaz Faramarzi ( $\nabla$ elnazfaramarzi849@gmail.com )

Liver and Gastrointestinal Diseases Research Center, Tabriz University of Medical Sciences https://orcid.org/0000-0003-4128-433X

\section{Research}

Keywords: Cardiometabolic phenotype, uric acid, cohort study, metabolic syndrome

Posted Date: March 15th, 2021

DOl: https://doi.org/10.21203/rs.3.rs-291727/v1

License: (c) (i) This work is licensed under a Creative Commons Attribution 4.0 International License. Read Full License 


\section{Abstract}

Background: As of now, it is unknown whether hyperuricemia can be considered as an independent risk factor or just as a marker to represent the correlation between uric acid and other metabolic syndrome (MetS) risk factors. To the best of the authors' knowledge, no other work has been reported to study this relationship between serum uric acid (SUA) and cardiometabolic phenotypes. In this work, we intend to study the correlation between SUA and the cardiometabolic phenotype among healthcare workers in Azar cohort study.

Method: In this cross-sectional study, anthropometric measurements, serum fasting blood sugar (FBS), triglyceride (TG), cholesterol, high lipoprotein density (HDL), liver enzymes, blood urea nitrogen (BUN), SUA, creatinine ( $\mathrm{Cr}$ ), and blood pressures of 1458 healthcare workers were evaluated. MetS was diagnosed based on ATP III. We classified the participants into four cardiometabolic phenotypes, i.e., metabolically-healthy lean (MHL), metabolically-unhealthy lean (MUHL), metabolically-healthy obese (MHO), and metabolically-unhealthy obese (MUHO).

Results: MHL (32.6\%) and MHO (66\%) have the highest prevalence rate in the first and second SUA tertiles, respectively, which are statistically significant (P-value $\leq 0.001)$. We observed an ascending trend in the mean values of WC, TG, cholesterol, low HDL, FBS, BUN, Cr, SBP, DBP, BMI, and liver enzymes from the first SUA tertile to the third SUA tertile (P-value $<0.05$ ). Compared to the lowest SUA tertile, the odds of $\mathrm{MHO}$ and $\mathrm{MUHO}$ increased by $2.29(95 \% \mathrm{Cl} 1.46-3.59)$ and $5.38(95 \% \mathrm{Cl} 3.45-9.39)$, respectively. In contrast, no similar trend was observed regarding the association between MUHL and the SUA tertile.

Conclusion: We proposed the use of the easily-measured SUA level as a marker for early diagnosis of atrisk MUHL and $\mathrm{MHO}$ individuals to administer proper interventions. Further prospective works are needed to identify the effects of SUA on the progression of MetS in various body size subgroups.

\section{Introduction}

One of the growing and escalating health challenges of the twenty-first century is the metabolic syndrome (MetS) with an increasing prevalence in both developed and developing countries [1]. In African and Asian countries, MetS prevalence ranges from 16.3 to 33.4\% [2], while in Tehran, 33.7\% of the adult population have MetS, as reported in Tehran Lipid and Glucose Study (TLGS) [3]. MetS can predict the risk of developing diabetes, chronic kidney disease, cardiovascular diseases, and degenerative diseases in older people [1]. However, understanding the pathogenesis of MetS, including its underlying mechanisms, the development sequence, and the interactions among individual components, is a very complex task [1].

MetS has several risk factors, such as hypertriglyceridemia, central obesity, hypertension, and insulin resistance, which can be accompanied by an increase in uric acid level, a pro-inflammatory agent that affects endothelial cells $[4,5]$. Increased production or decreased excretion of uric acid can lead to hyperuricemia, which is a metabolic syndrome caused by purine metabolic abnormalities [6]. 
Several studies have revealed the relationship between urate, MetS, and its components $[7,8]$. Moreover, a correlation between body mass index (BMI) and circulating urate concentrations has been reported. Individuals classified in the high BMI category show high uric acid levels, which are highly associated with the metabolic syndrome $[9,10]$. It has been shown that the genetic predisposition of people with high uric acid levels is associated with blood pressure elevation and dyslipidemia, but not with obesity/diabetes, all of which are components of MetS. This may suggest that high serum uric acid (SUA) may involve a separate pathway for the development of MetS, independent of obesity [1].

In this regard, despite the fact that obesity has been documented as a major risk factor for MetS, some individuals identified as obese may not display any signs of typical metabolic disorders, and they may have a lower risk of obesity-related complications. According to the obtained data, $10 \%-25 \%$ of obese people can be classified as metabolically-healthy obese ( $\mathrm{MHO})$. According to the results of the study carried out by Velho et al., the prevalence of MHO varies from 3.3 to $32.1 \%$ in men and from $11.4-43.3 \%$ in women [11]. The findings of various studies suggest that $\mathrm{MHO}$ subjects have more dangerous conditions than metabolically-healthy subjects because of the higher risk for developing hypertension, type 2 diabetes, and the metabolic syndrome. In addition, a study on normal-weight adults (body mass index $[\mathrm{BMI}]<25.0 \mathrm{~kg} / \mathrm{m}^{2}$ ) living in the United States shows that $24 \%$ of the adults are metabolically abnormal. Abnormal metabolic conditions predispose this group to chronic diseases compared to metabolically-healthy normal-weight individuals [12].

However, the exact metabolic biomarkers that cause healthy individuals to become metabolically unhealthy during their lifetime are not fully understood. We are hypothesizing that the elevated SUA may play a role in the pathogenesis of MetS in metabolically-unhealthy lean (MUHL) or metabolicallyunhealthy obese (MUHO) individuals. As of now, it is unknown whether hyperuricemia can be considered as an independent risk factor or just as a marker to represent the correlation between uric acid and other MetS risk factors. To the best of the authors' knowledge, no other work has been reported to study this relationship between SUA and cardiometabolic phenotypes. In this work, we intend to study the correlation between SUA and the cardiometabolic phenotype among healthcare workers in Azar cohort study.

\section{Materials And Methods}

We studied a cohort that is a part of a large prospective epidemiological researches in Iran (the Persian cohort study)[13]. The cohort study on the healthcare workers was carried out in 2020 as a part of the Azar cohort study, which was conducted by the liver and Gastrointestinal diseases Research center of Tabriz University of medical sciences[14]. The purpose of this cohort study was to evaluate 6000 participants who were related to Tabriz University of Medical Sciences (TUMS), including healthcare employees in hospitals, schools, and district health networks. This study intended to characterize the risk factors of non-communicable diseases (NCD) among healthcare providers, official staff, and professors of Tabriz University of Medical Sciences. 
Our baseline assessment consisted of a face-to-face health interview or a health examination in terms of a broad range of established and novel risk factors of NCDs.

Data from a total of 1458 participants were used for this cohort study. All involved participants provided written informed consent, and the study was approved by the Ethics Committee of Tabriz University of Medical Sciences (IR.TBZMED.REC.1396.1263).

Participants of this study include fulltime and long-term contract employees aged between 18 and 75 years who are not pregnant or lactating, and who are not planning to retire within the next five years. Patients who reported having been diagnosed with debilitating psychiatric disorders or physical illnesses by a health professional were excluded from this study

\subsection{Demographic Characteristics of the Participants}

We used a questionnaire for evaluating demographic characteristics, such as age, gender, marital status, and educational level. Moreover, lifestyle patterns, i.e., smoking, drug use, hookah use, alcohol consumption, and being a passive smoker were also assessed by the questionnaire.

\subsection{Anthropometric and Blood Pressure Measurements}

Bodyweight, height, and waist circumference of all the subjects were measured, and their body mass index was determined using the standard formula, i.e., weight $(\mathrm{kg}) /$ height $^{2}(\mathrm{~m})$. The anthropometric measurements are described in detail elsewhere [14]. Blood pressure was measured by a trained nurse twice with a two-minute interval, and twice in each arm in a sitting position after 10 minutes of rest by using a mercury sphygmomanometer (Rudolf Richter, DE-72417, Germany). The average values were calculated and used in the analysis as the systolic and diastolic blood pressure.

\subsection{Biochemical Factors}

After an overnight fast ( $\geq 12$ hours), blood samples were obtained from the participants to determine serum uric acid (SUA), fasting blood sugar (FBS), triglyceride (TG), high-density lipoprotein (HDL), cholesterol, aspartate aminotransferase (AST), alanine aminotransferase, alkaline phosphatase, gammaglutamyl transferase (GGT), blood urea nitrogen (BUN), and creatinine using enzymatic methods. In addition, low-density lipoprotein (LDL) levels were calculated based on the Friedewald equation.

\subsection{Definition of MetS}

The Adult Treatment Panel III (ATP III) of the National Cholesterol Education Program defines individuals with MetS as subjects who meet three or more of the following conditions: hypertension, defined as a systolic blood pressure $\geq 130$ and/or a diastolic blood pressure $\geq 85 \mathrm{mmHg}$, or subjects using antihypertensive medication; waist circumference $\geq 102 \mathrm{~cm}$ in men and $\geq 88 \mathrm{~cm}$ in women; hypertriglyceridemia, defined as $T G \geq 150 \mathrm{mg} / \mathrm{dl}$, or individuals treated for elevated triglycerides; low $\mathrm{HDL}-\mathrm{C}$ values $<40 \mathrm{mg} / \mathrm{dl}$ in men and $<50 \mathrm{mg} / \mathrm{dl}$ in women; and high fasting glucose $\geq 100 \mathrm{mg} / \mathrm{dl}$, or the use of glucose-lowering medication [15]. 
In this paper, subjects were divided into four cardiometabolic phenotypes based on the BMI cutoff point $\left(25 \mathrm{~kg} / \mathrm{m}^{2}\right)$, and the presence or absence of MetS. The four phenotypes include MUHO, i.e., metabolicallyunhealthy obese (MetS present and $\mathrm{BMI} \geq 25 \mathrm{~kg} / \mathrm{m}^{2}$ ); MUHL, i.e., metabolically-unhealthy lean (MetS present and $\mathrm{BMI}<25 \mathrm{~kg} / \mathrm{m}^{2}$ ); MHO, i.e., metabolically-healthy obese (no MetS and BMI $\geq 25 \mathrm{~kg} / \mathrm{m}^{2}$ ); and $\mathrm{MHL}$, i.e., metabolically-healthy lean (no MetS and $\mathrm{BMI}<25 \mathrm{~kg} / \mathrm{m}^{2}$ ).

\subsection{Statistical Analysis}

Statistical analysis was performed using IBM SPSS Statistics version 11.5 (IBM, Chicago, IL). Continuous variables were expressed as mean \pm standard deviation, and their differences were assessed using a chisquare analysis across the four study groups. Categorical variables were presented as numbers (percentages), and their differences among the four groups were measured using one-way analysis of variance (ANOVA).

The participants were divided into three tertiles based on serum SUA, i.e., Tertile 1: $2-4 \mathrm{mg} / \mathrm{dl}$; Tertile 2: 4.1-5.1 mg/dl; and Tertile 3: 5.2-10 mg/dl.

Multinomial logistic regression analysis was performed to determine the relationship between cardiometabolic phenotype and serum SUA tertile. Moreover, crude and adjusted odds ratios (OR) and their corresponding $95 \%$ confidence intervals $(95 \% \mathrm{Cl})$ were calculated. We perform the analysis after adjusting for confounding factors, including age, gender, educational level, marital status, and current smoking status, while MHL was used as a reference group.

We considered $\mathrm{BMI}$ as the basis for classification, and seven underweight interviewees were excluded. Eventually, statistical analysis was carried out on 1451 subjects. $P$ values $<0.05$ were considered statistically significant.

\section{Results}

Table 1 presents the baseline characteristics of the participants for individual SUA tertiles. The third tertile includes a higher percentage of male and married participants than the first two tertiles (P-value $<0.001)$. Moreover, MHL (32.6\%) and MHO (66\%) have the greatest prevalence in the first and second SUA tertiles, which are statistically significant (P-value $\leq 0.001$ ). We observed an ascending trend in the mean values of WC, TG, cholesterol, low HDL, FBS, BUN, Cr, SBP, DBP, BMI, and liver enzymes from the first to the third SUA tertile (P-value < 0.05). 
Table 1

Baseline characteristics of participants according to uric acid tertile $(n=1451)$

Serum uric aicd

(mg/dl)

Tertile $1=2-\quad$ Tertile2 $=4.1-5.1$

4

$(n=518)$
Tertile3 $=5.2-\quad P$ value 10

$(n=483)$

\section{Gender $n(\%)$}

Male

Female

Education level $\mathrm{n}(\%)$

Illiterate

Primary school

High school

University

Marital status $\mathrm{n}(\%)$

Not married

Married

Cardiometabolic phenotype $n$

(\%)

${ }^{*} \mathrm{MHL}$

169(32.6)

88(19.6)

61(12.6)

${ }^{* \star} \mathrm{MUHL}$

$6(1.2)$

$3(0.7)$

$3(0.6)$

${ }^{\mathrm{MHO}}$

291(56.2)

297(66)

62(13.8)

$318(65.8)$

$52(10)$

$25(5.6)$

$31(6.4)$

91(18.8)

352(72.9)

'MUHO

\section{Smoking status $\mathrm{n}(\%)$}

Yes

No

\section{Alcohol consumption $\mathrm{n}(\%)$}

${ }^{€} \mathrm{P}$ value :chi square test ; $;$ P- value :one way Anova ; ${ }^{*} \mathrm{MHL}$ : metabolically healthy lean $;{ }^{* \star} \mathrm{MUHL}$ : metabolically unhealthy lean ; $\mathrm{MHO}$ : Metabolically healthy obese; ${ }^{\mathbf{9 4}} \mathrm{MUHO}$ : metabolically unhealthy obese 


\begin{tabular}{|c|c|c|c|c|}
\hline & & $\begin{array}{l}\text { Serum uric aicd } \\
(\mathrm{mg} / \mathrm{dl})\end{array}$ & & \\
\hline Yes & $1(0.2)$ & $5(1.1)$ & $2(0.4)$ & \\
\hline No & $513(99.8)$ & $441(98.9)$ & $480(99.6)$ & \\
\hline Drug use $n(\%)$ & & & & 0.64 \\
\hline Yes & $1(0.2)$ & $0(0)$ & $0(0)$ & \\
\hline \multirow[t]{2}{*}{ No } & $513(99.8)$ & $446(100)$ & $482(100)$ & \\
\hline & mean $\pm S D$ & mean \pm SD & mean $\pm S D$ & \\
\hline Age (year) & $42.25 \pm 6.47$ & $42.49 \pm 6.72$ & $42.88 \pm 6.97$ & $¥ 0.33$ \\
\hline Waist circumference $(\mathrm{cm})$ & $92.96 \pm 9.01$ & $95.33 \pm 8.29$ & $98.06 \pm 9.19$ & $\begin{array}{l}¥ \\
<0.001\end{array}$ \\
\hline Triglyceride (mg/dl) & $\begin{array}{l}100.14 \pm \\
41.16\end{array}$ & $115.47 \pm 54.99$ & $149.64 \pm 74.58$ & $\begin{array}{l}¥ \\
<0.001\end{array}$ \\
\hline Fasting blood sugar (mg/dl) & $\begin{array}{l}85.37 \pm \\
23.92\end{array}$ & $85.44 \pm 17.90$ & $88.32 \pm 17.61$ & $¥ 0.03$ \\
\hline Blood urea nitrogen $(\mathrm{mg} / \mathrm{dl})$ & $11.78 \pm 3.34$ & $12.46 \pm 3.11$ & $13.31 \pm 3.16$ & $\begin{array}{l}¥ \\
<0.001\end{array}$ \\
\hline Creatinine (mg/dl) & $0.93 \pm 0.14$ & $1.01 \pm 0.14$ & $1.10 \pm 0.17$ & $\begin{array}{l}¥ \\
<0.001\end{array}$ \\
\hline $\begin{array}{l}\text { High density lipoprotein } \\
(\mathrm{mg} / \mathrm{dl})\end{array}$ & $\begin{array}{l}48.98 \pm \\
10.83\end{array}$ & $45.86 \pm 10.62$ & $42.59 \pm 9.12$ & $\begin{array}{l}¥ \\
<0.001\end{array}$ \\
\hline Cholesterol (mg/dl) & $\begin{array}{l}162.28 \pm \\
35.66\end{array}$ & $163.97 \pm 35.64$ & $176.61 \pm 37.68$ & $\begin{array}{l}¥ \\
<0.001\end{array}$ \\
\hline Low density lipoprotein (mg/dl) & $\begin{array}{l}93.27 \pm \\
29.78\end{array}$ & $95.30 \pm 29.19$ & $104.55 \pm 30.29$ & $\begin{array}{l}¥ \\
<0.001\end{array}$ \\
\hline $\begin{array}{l}\text { Systolic blood pressure (mm } \\
\mathrm{Hg})\end{array}$ & $\begin{array}{l}105.89 \pm \\
12.95\end{array}$ & $111.13 \pm 15.26$ & $115.81 \pm 13.55$ & $\begin{array}{l}¥ \\
<0.001\end{array}$ \\
\hline $\begin{array}{l}\text { Diastolic blood pressure }(\mathrm{mm} \\
\mathrm{Hg})\end{array}$ & $73.24 \pm 8.35$ & $76.29 \pm 9.57$ & $79.18 \pm 8.85$ & $\begin{array}{l}¥ \\
<0.001\end{array}$ \\
\hline Body mass index $\left(\mathrm{kg} / \mathrm{m}^{2}\right)$ & $26.94 \pm 4.09$ & $27.95 \pm 3.87$ & $28.53 \pm 3.77$ & $\begin{array}{l}¥ \\
<0.001\end{array}$ \\
\hline $\begin{array}{l}€_{\mathrm{P}} \text { value :chi square test } ; \stackrel{*}{\mathrm{P}} \text { - va } \\
\text { metabolically unhealthy lean } ;{ }^{\bullet} \wedge \\
\text { obese }\end{array}$ & $\begin{array}{l}\text { one way An } \\
\text { : Metabolic }\end{array}$ & $\begin{array}{l}{ }^{*} \mathrm{MHL}: \text { metabc } \\
\text { ealthy obese }\end{array}$ & $\begin{array}{l}\text { ealthy lean } ;^{* *} \mathrm{M} \\
\text { metabolically }\end{array}$ & $\begin{array}{l}\text { L: } \\
\text { ealthy }\end{array}$ \\
\hline
\end{tabular}




\begin{tabular}{|c|c|c|c|c|}
\hline & & $\begin{array}{l}\text { Serum uric aicd } \\
(\mathrm{mg} / \mathrm{dl})\end{array}$ & & \\
\hline $\begin{array}{l}\text { Aspartate aminotransferase } \\
\text { (IU/I) }\end{array}$ & $17.23 \pm 6.79$ & $19.65 \pm 6.76$ & $23.77 \pm 10.32$ & $\begin{array}{l}¥ \\
<0.001\end{array}$ \\
\hline Alanin amino transferase (IU/I) & $\begin{array}{l}18.11 \pm \\
10.84\end{array}$ & $23.48 \pm 13.22$ & $33.67 \pm 21.66$ & $\begin{array}{l}¥ \\
<0.001\end{array}$ \\
\hline Alkaline phosphatase (IU/I) & $\begin{array}{l}160.56 \pm \\
45.43\end{array}$ & $179.12 \pm 53.21$ & $188.04 \pm 53.02$ & $\begin{array}{l}¥ \\
<0.001\end{array}$ \\
\hline $\begin{array}{l}\text { Gamma glutamyle transferase } \\
(\mathrm{IU} / \mathrm{I})\end{array}$ & $\begin{array}{l}18.24 \pm \\
18.05\end{array}$ & $22.24 \pm 14.13$ & $29.82 \pm 18.07$ & $\begin{array}{l}¥ \\
<0.001\end{array}$ \\
\hline \multicolumn{5}{|c|}{$\begin{array}{l}{ }^{€} \mathrm{P} \text { value :chi square test ; }{ }^{*} \mathrm{P} \text { - value :one way Anova ; }{ }^{*} \mathrm{MHL} \text { : metabolically healthy lean } ;{ }^{* *} \mathrm{MUHL} \text { : } \\
\text { metabolically unhealthy lean ; }{ }^{4} \mathrm{MHO} \text { : Metabolically healthy obese } ;{ }^{49} \mathrm{MUHO} \text { : metabolically unhealthy } \\
\text { obese }\end{array}$} \\
\hline
\end{tabular}

Table 2 shows that in all cardiometabolic phenotype classes, the lowest $(P$-value $<0.001)$ proportion of females as dose-dependent of SUA was in the third tertile. 
Table 2

Demographic, anthropometric and biochemical factors according to serum uric acid tertile stratified by cardiometabolic phenotype

Serum Uric acid level

$(\mathrm{mg} / \mathrm{dl})$

$\begin{array}{llll}\text { Tertile } 1= & \text { Tertile2 }=4.1-5.1 & \text { Tertile3 }= & \text { P value } \\ 2-4 & (n=450) & 5.2-10 & \\ (n=518) & & (n=483)\end{array}$

$\mathrm{MHL}$

Gender $\mathrm{n}(\%)$

$€$

$<0.001$

Male

$50(29.6)$

$59(67)$

$55(90.2)$

Female

119(70.4)

29(33)

mean \pm SD

$41.38 \pm 6.81$

$41.26 \pm 6.58$

$86.92 \pm 5.57$

$100.64 \pm 50.37$

$89.73 \pm$

31.82

Fasting blood sugar $(\mathrm{mg} / \mathrm{dl}) \quad 83.09 \pm$

15.52

Blood urea nitrogen $(\mathrm{mg} / \mathrm{dl})$

$11.47 \pm 3.22$

$12.80 \pm 3.46$

$1.03 \pm 0.15$

$0.95 \pm 0.14$

$81.93 \pm 8.29$

0

$45.93 \pm 9.94$

$50.40 \pm$

11.07

$157.42 \pm 35$.

61

Cholesterol (mg/dl)

$89.06 \pm$ 29.82

$(\mathrm{mg} / \mathrm{dl})$

Aspartate aminotransferase (IU/I)

Alanin amino transferase $(\mathrm{IU} / \mathrm{I})$
$16.56 \pm 4.76 \quad 19.80 \pm 7.95$

$16.48 \pm 8.58$

$21.77 \pm 12.65$
6(9.8)

mean $\pm S D$

$41.11 \pm 7.48 \quad ¥ 0.97$

$86.66 \pm 5.53 \quad ¥ 0.17$

$113.31 \pm \quad ¥$

41.45

$<0.001$

$83.24 \pm 8.54 \quad ¥ 0.75$

$13.04 \pm 2.87 \quad ¥ 0.006$

$1.13 \pm 0.17 \quad ¥$

$<0.001$

$44.30 \pm 8.77 \quad ¥$

$<0.001$

$166.24 \pm$

28.76

$¥ 0.13$

$99.29 \pm 23.99 \quad ¥ 0.04$

$21.49 \pm 10.12 \quad ¥ \quad{ }_{0} 0.001$

$26.52 \pm 15.48 \quad ¥_{<}<0.001$

${ }^{€} \mathrm{P}$ value :chi square test ; ${ }^{*} \mathrm{P}$ - value :one way ANOVA ; ${ }^{*} \mathrm{MHL}$ : metabolically healthy lean $;{ }^{* *} \mathrm{MUHL}$ : metabolically unhealthy lean ; $\mathbf{M H O}$ : Metabolically healthy obese; ; ${ }^{\mathbf{9}} \mathrm{MUHO}$ : metabolically unhealthy obese 


\section{Serum Uric acid level $(\mathrm{mg} / \mathrm{dl})$}

\begin{tabular}{|c|c|c|c|c|}
\hline Alkaline phosphatase (IU/I) & $\begin{array}{l}157.0 \pm \\
42.88\end{array}$ & $170.70 \pm 42.87$ & $\begin{array}{l}182.96 \pm \\
48.24\end{array}$ & $¥_{<0}<001$ \\
\hline $\begin{array}{l}\text { Gamma glutamyle } \\
\text { transferase (IU/I) }\end{array}$ & $15.77 \pm 9.88$ & $18.60 \pm 8.75$ & $21.81 \pm 8.52$ & $¥<0.001$ \\
\hline $\begin{array}{l}\text { Systolic blood pressure (mm } \\
\mathrm{Hg})\end{array}$ & $\begin{array}{l}102.71 \pm \\
11.71\end{array}$ & $107.02 \pm 14.11$ & $\begin{array}{l}109.95 \pm \\
12.38\end{array}$ & $\begin{array}{l}¥ \\
<0.001\end{array}$ \\
\hline $\begin{array}{l}\text { Diastolic blood pressure }(\mathrm{mm} \\
\mathrm{Hg})\end{array}$ & $71.24 \pm 7.22$ & $73.01 \pm 7.97$ & $74.83 \pm 7.28$ & $¥ 0.004$ \\
\hline \multicolumn{5}{|l|}{ MUHL } \\
\hline Gender $\mathrm{n}(\%)$ & & & & $€_{0.01}$ \\
\hline Male & $0(0)$ & $2(66.7)$ & $3(100)$ & \\
\hline \multirow[t]{2}{*}{ Female } & $6(100)$ & $1(33.3)$ & $0(0)$ & \\
\hline & mean \pm SD & mean \pm SD & mean $\pm S D$ & \\
\hline Age (year) & $46.83 \pm 6.70$ & $40.33 \pm 2.88$ & $43.67 \pm 7.57$ & $¥ 0.37$ \\
\hline Waist circumference $(\mathrm{cm})$ & $93.25 \pm 4.05$ & $92.13 \pm 3.66$ & $85.23 \pm 3.30$ & $¥ 0.04$ \\
\hline Triglyceride (mg/dl) & $131 \pm 54.33$ & $232.33 \pm 105.07$ & $\begin{array}{l}215.33 \pm \\
43.98\end{array}$ & $¥ 0.10$ \\
\hline Fasting blood sugar (mg/dl) & $93.5 \pm 38.35$ & $75.33 \pm 2.51$ & $90.0 \pm 14.79$ & $¥ 0.68$ \\
\hline Blood urea nitrogen $(\mathrm{mg} / \mathrm{dl})$ & $11.16 \pm 2.63$ & $16.33 \pm 4.72$ & $12.66 \pm 2.30$ & $¥ 0.12$ \\
\hline Creatinine $(\mathrm{mg} / \mathrm{dl})$ & $0.84 \pm 0.12$ & $0.96 \pm 0.12$ & $1.03 \pm 0.24$ & $¥ 0.25$ \\
\hline $\begin{array}{l}\text { High density lipoprotein } \\
(\mathrm{mg} / \mathrm{dl})\end{array}$ & $42.17 \pm 4.87$ & $38 \pm 5.19$ & $41 \pm 7.93$ & $¥ 0.60$ \\
\hline Cholesterol (mg/dl) & $155 \pm 26.48$ & $185.33 \pm 36.85$ & $\begin{array}{l}163.33 \pm \\
44.06\end{array}$ & $¥ 0.47$ \\
\hline $\begin{array}{l}\text { Low density lipoprotein } \\
(\mathrm{mg} / \mathrm{dl})\end{array}$ & $86.66 \pm 21.5$ & $100.66 \pm 44.61$ & $79.33 \pm 40.67$ & $¥ 0.72$ \\
\hline $\begin{array}{l}\text { Aspartate aminotransferase } \\
\text { (IU/I) }\end{array}$ & $31.5 \pm 37.08$ & $15 \pm 1.73$ & $23 \pm 6.24$ & $¥ 0.70$ \\
\hline
\end{tabular}

$€ \mathrm{P}$ value :chi square test; ${ }^{*} \mathrm{P}$ - value :one way ANOVA ; ${ }^{*} \mathrm{MHL}$ : metabolically healthy lean $;^{* *} \mathrm{MUHL}$ : metabolically unhealthy lean; ${ }^{\mathbf{9}} \mathrm{MHO}$ : Metabolically healthy obese ; ${ }^{\mathbf{9 4}} \mathrm{MUHO}$ : metabolically unhealthy obese 


\section{Serum Uric acid level $(\mathrm{mg} / \mathrm{dl})$}

\begin{tabular}{|c|c|c|c|c|}
\hline $\begin{array}{l}\text { Alanin amino transferase } \\
(\mathrm{IU} / \mathrm{I})\end{array}$ & $\begin{array}{l}34.83 \pm \\
42.74\end{array}$ & $16 \pm 3.46$ & $29 \pm 19.62$ & $¥ 0.73$ \\
\hline Alkaline phosphatase (IU/I) & $\begin{array}{l}135.83 \pm \\
19.71\end{array}$ & $185.66 \pm 60.91$ & $\begin{array}{l}190.66 \pm \\
62.42\end{array}$ & $¥ 0.17$ \\
\hline $\begin{array}{l}\text { Gamma glutamyle } \\
\text { transferase }(\mathrm{IU} / \mathrm{l})\end{array}$ & $\begin{array}{l}31.66 \pm \\
40.69\end{array}$ & $17 \pm 5$ & $20 \pm 2$ & $¥ 0.75$ \\
\hline $\begin{array}{l}\text { Systolic blood pressure (mm } \\
\mathrm{Hg})\end{array}$ & $\begin{array}{l}106.27 \pm \\
10.63\end{array}$ & $111.33 \pm 14.94$ & $135.33 \pm 6.02$ & $¥ 0.01$ \\
\hline $\begin{array}{l}\text { Diastolic blood pressure (mm } \\
\mathrm{Hg})\end{array}$ & $\begin{array}{l}71.27 \pm \\
11.63\end{array}$ & $84.22 \pm 13.84$ & $87.55 \pm 7.04$ & $¥ 0.13$ \\
\hline \multicolumn{5}{|l|}{$\mathrm{MHO}$} \\
\hline Gender $n(\%)$ & & & & $€_{<0.001}$ \\
\hline Male & $47(16.2)$ & $128(43.1)$ & $266(83.6)$ & \\
\hline \multirow[t]{2}{*}{ Female } & $244(83.8)$ & $169(56.9)$ & $52(16.4)$ & \\
\hline & mean \pm SD & mean $\pm S D$ & mean \pm SD & \\
\hline Age (year) & $42.29 \pm 6.18$ & $42.26 \pm 6.56$ & $43.03 \pm 7.03$ & $¥ 0.26$ \\
\hline Waist circumference $(\mathrm{cm})$ & $95.73 \pm 7.81$ & $96.35 \pm 7.01$ & $98.47 \pm 8.11$ & $¥ 0.003$ \\
\hline Triglyceride (mg/dl) & $\begin{array}{l}96.22 \pm \\
31.41\end{array}$ & $106.82 \pm 39.69$ & $\begin{array}{l}137.58 \pm \\
64.92\end{array}$ & $¥<0.001$ \\
\hline Fasting blood sugar (mg/dl) & $\begin{array}{l}82.42 \pm \\
19.69\end{array}$ & $83.20 \pm 10.49$ & $86.14 \pm 10.50$ & $¥<0.001$ \\
\hline Blood urea nitrogen $(\mathrm{mg} / \mathrm{dl})$ & $11.79 \pm 3.39$ & $12.27 \pm 2.99$ & $13.64 \pm 3.22$ & $¥<0.001$ \\
\hline Creatinine (mg/dl) & $0.93 \pm 0.13$ & $1 \pm 0.13$ & $1.11 \pm 0.17$ & $¥<0.001$ \\
\hline $\begin{array}{l}\text { High density lipoprotein } \\
(\mathrm{mg} / \mathrm{dl})\end{array}$ & $\begin{array}{l}49.75 \pm \\
10.49\end{array}$ & $46.75 \pm 11.06$ & $43.44 \pm 9.16$ & $¥<0.001$ \\
\hline Cholesterol (mg/dl) & $\begin{array}{l}165.17 \pm \\
34.82\end{array}$ & $163.80 \pm 35.79$ & $\begin{array}{l}176.44 \pm \\
38.24\end{array}$ & $¥<0.001$ \\
\hline $\begin{array}{l}\text { Low density lipoprotein } \\
(\mathrm{mg} / \mathrm{dl})\end{array}$ & $\begin{array}{l}96.16 \pm \\
29.22\end{array}$ & $95.65 \pm 29.12$ & $\begin{array}{l}105.81 \pm \\
31.13\end{array}$ & $¥<0.001$ \\
\hline
\end{tabular}

$€_{\mathrm{P}}$ value :chi square test ; ${ }^{*} \mathrm{P}$ - value :one way ANOVA ; ${ }^{*} \mathrm{MHL}$ : metabolically healthy lean ${ }^{* *} \mathrm{MUHL}$ : metabolically unhealthy lean ; $\mathrm{MHO}$ : Metabolically healthy obese ; ${ }^{\mathbf{9 4}} \mathrm{MUHO}$ : metabolically unhealthy obese 


\section{Serum Uric acid level $(\mathrm{mg} / \mathrm{dl})$}

\begin{tabular}{|c|c|c|c|c|}
\hline $\begin{array}{l}\text { Aspartate aminotransferase } \\
(\mathrm{IU} / \mathrm{I})\end{array}$ & $17.19 \pm 5.62$ & $19.39 \pm 6.29$ & $23.60 \pm 9.71$ & $¥<0.001$ \\
\hline $\begin{array}{l}\text { Alanin amino transferase } \\
(\mathrm{IU} / \mathrm{I})\end{array}$ & $18.23 \pm 9.87$ & $23.05 \pm 12.84$ & $32.29 \pm 20.67$ & $¥<0.001$ \\
\hline Alkaline phosphatase (IU/I) & $\begin{array}{l}161.93 \pm \\
47.30\end{array}$ & $179.29 \pm 54.64$ & $\begin{array}{l}188.41 \pm \\
50.95\end{array}$ & $¥<0.001$ \\
\hline $\begin{array}{l}\text { Gamma glutamyle } \\
\text { transferase }(\mathrm{IU} / \mathrm{l})\end{array}$ & $\begin{array}{l}18.67 \pm \\
20.82\end{array}$ & $21.91 \pm 14.24$ & $30.22 \pm 18.80$ & $¥<0.001$ \\
\hline $\begin{array}{l}\text { Systolic blood pressure (mm } \\
\mathrm{Hg})\end{array}$ & $\begin{array}{l}105.89 \pm \\
12.38\end{array}$ & $110.25 \pm 15.01$ & $\begin{array}{l}110.41 \pm \\
12.53\end{array}$ & $¥<0.001$ \\
\hline $\begin{array}{l}\text { Diastolic blood pressure }(\mathrm{mm} \\
\mathrm{Hg})\end{array}$ & $73.32 \pm 8.33$ & $76.10 \pm 9.57$ & $78.48 \pm 8.52$ & $¥<0.001$ \\
\hline \multicolumn{5}{|l|}{ MUHO } \\
\hline Gender $n(\%)$ & & & & $€_{<0.001}$ \\
\hline Male & $15(28.8)$ & $30(48.4)$ & $70(69.3)$ & \\
\hline \multirow[t]{2}{*}{ Female } & 37(71.2.) & $32(86.9)$ & $31(30.7)$ & \\
\hline & mean $\pm S D$ & mean $\pm S D$ & mean $\pm S D$ & \\
\hline Age (year) & $44.71 \pm 6.91$ & $45.27 \pm 6.80$ & $43.45 \pm 6.36$ & ${ }^{¥} 0.2$ \\
\hline Waist circumference $(\mathrm{cm})$ & $\begin{array}{l}101.31 \pm \\
8.22\end{array}$ & $102.52 \pm 7.97$ & $104.04 \pm 7.73$ & $¥ 0.27$ \\
\hline Triglyceride (mg/dl) & $\begin{array}{l}151.96 \pm \\
68.16\end{array}$ & $172.33 \pm 77.40$ & $\begin{array}{l}207.60 \pm \\
87.14\end{array}$ & $¥<0.001$ \\
\hline Fasting blood sugar (mg/dl) & $\begin{array}{l}108.34 \pm \\
45.24\end{array}$ & $101.62 \pm 37.60$ & $98.19 \pm 31.10$ & $¥ 0.27$ \\
\hline Blood urea nitrogen $(\mathrm{mg} / \mathrm{dl})$ & $11.96 \pm 3.61$ & $12.65 \pm 2.98$ & $12.46 \pm 3.03$ & $¥ 0.49$ \\
\hline Creatinine (mg/dl) & $0.94 \pm 0.15$ & $1.04 \pm 0.14$ & $1.09 \pm 0.16$ & $\begin{array}{l}¥ \\
<0.001\end{array}$ \\
\hline $\begin{array}{l}\text { High density lipoprotein } \\
(\mathrm{mg} / \mathrm{dl})\end{array}$ & $40.85 \pm 8.59$ & $41.82 \pm 8.47$ & $38.90 \pm 8.34$ & $¥ 0.08$ \\
\hline Cholesterol (mg/dl) & $\begin{array}{l}162.78 \pm \\
40.22\end{array}$ & $175.82 \pm 34.64$ & $\begin{array}{l}183.83 \pm \\
39.36\end{array}$ & $¥ 0.05$ \\
\hline
\end{tabular}

$€_{\mathrm{P}}$ value :chi square test ; ${ }^{*} \mathrm{P}$ - value :one way ANOVA ; ${ }^{*} \mathrm{MHL}$ : metabolically healthy lean ${ }^{* *} \mathrm{MUHL}$ : metabolically unhealthy lean ; $\mathrm{MHO}$ : Metabolically healthy obese ; ${ }^{\mathbf{9 4}} \mathrm{MUHO}$ : metabolically unhealthy obese 


\begin{tabular}{|c|c|c|c|c|}
\hline & & $\begin{array}{l}\text { Serum Uric acid level } \\
(\mathrm{mg} / \mathrm{dl})\end{array}$ & & \\
\hline $\begin{array}{l}\text { Low density lipoprotein } \\
(\mathrm{mg} / \mathrm{dl})\end{array}$ & $\begin{array}{l}91.53 \pm \\
32.32\end{array}$ & $101.72 \pm 31.26$ & $\begin{array}{l}104.52 \pm \\
30.49\end{array}$ & $¥ 0.006$ \\
\hline $\begin{array}{l}\text { Aspartate aminotransferase } \\
(\mathrm{IU} / \mathrm{I})\end{array}$ & $17.96 \pm 7.22$ & $20.90 \pm 7.13$ & $25.74 \pm 12.04$ & $¥<0.001$ \\
\hline $\begin{array}{l}\text { Alanin amino transferase } \\
(\mathrm{IU} / \mathrm{I})\end{array}$ & $\begin{array}{l}20.86 \pm \\
12.91\end{array}$ & $28.33 \pm 15.07$ & $39.29 \pm 26.29$ & $¥<0.001$ \\
\hline Alkaline phosphatase (IU/I) & $\begin{array}{l}167.30 \pm \\
43.98\end{array}$ & $189.90 \pm 58.63$ & $\begin{array}{l}189.86 \pm \\
61.88\end{array}$ & $¥ 0.04$ \\
\hline $\begin{array}{l}\text { Gamma glutamyle } \\
\text { transferase (IU/I) }\end{array}$ & $\begin{array}{l}22.30 \pm \\
17.25\end{array}$ & $29.25 \pm 17.46$ & $33.69 \pm 18.75$ & $¥ 0.05$ \\
\hline $\begin{array}{l}\text { Systolic blood pressure ( } \mathrm{mm} \\
\mathrm{Hg})\end{array}$ & $\begin{array}{l}116.35 \pm \\
14.89\end{array}$ & $121.15 \pm 14.13$ & $\begin{array}{l}122.27 \pm \\
14.70\end{array}$ & $¥<0.02$ \\
\hline $\begin{array}{l}\text { Diastolic blood pressure (mm } \\
\mathrm{Hg})\end{array}$ & $79.62 \pm 8.57$ & $81.47 \pm 9.33$ & $83.69 \pm 8.88$ & $¥ 0.001$ \\
\hline \multicolumn{5}{|c|}{$\begin{array}{l}\mathrm{E}_{\mathrm{P}} \text { value :chi square test } ;{ }^{*} \mathrm{P} \text { - value :one way ANOVA ; }{ }^{*} \mathrm{MHL} \text { : metabolically healthy lean } ;{ }^{* *} \mathrm{MUHL} \text { : } \\
\text { metabolically unhealthy lean ; } ; \mathrm{MHO} \text { : Metabolically healthy obese } ; ;^{\mathbf{9}} \mathrm{MUHO} \text { : metabolically unhealthy } \\
\text { obese }\end{array}$} \\
\hline
\end{tabular}

The average values for serum TG, WC, BUN, Cr, AST, ALT, ALP, and GGT in the MHL, MHO, and MUHO groups exhibited an elevated dose-response trend according to the SUA tertile ( $P$-value $<0.05)$. Additionally, similar to the trends for increasing SUA tertiles, the mean values for SBP and DBP have also increased markedly (P-value $<0.05)$.

In contrast to the other factors, WC, FBS, and cholesterol did not increase with increasing SUA. In addition, even WC in the MUHL group decreased noticeably based on SUA tertiles $(P=0.04)$. We present the relationship between SUA and cardiometabolic phenotype in Table 3. Our applied multinomial regression analysis indicates that compared to the lowest SUA tertile, the odds of MHO and MUHO increased by 2.29 (95\% $\mathrm{Cl} 1.46-3.59)$ and 5.38 (95\% $\mathrm{Cl} 3.45-9.39)$, respectively. After adjustment for different confounding factors (i.e., age, gender, marital status, education level, smoking, and alcohol consumption), the correlation was still significant. 
Table 3

Odds ratios and $95 \%$ confidence intervals for cardiometabolic phenotype according to serum uric acid Serum uric acid level $(\mathrm{mg} / \mathrm{dl})$

\begin{tabular}{|c|c|c|c|}
\hline & $\begin{array}{l}\text { Tertile } 1=2-4 \\
(n=518)\end{array}$ & $\begin{array}{l}\text { Tertile2 }=4.1-5.1 \\
(n=450)\end{array}$ & $\begin{array}{l}\text { Tertile3 }=5.2-10 \\
(n=483)\end{array}$ \\
\hline & $\mathrm{OR}(95 \% \mathrm{Cl})$ & $\mathrm{OR}(95 \% \mathrm{Cl})$ & $\mathrm{OR}(95 \% \mathrm{Cl})$ \\
\hline MUHL & Reference & $0.96(0.23-3.93)$ & $1.38(0.33-5.71)$ \\
\hline $\mathrm{MHO}$ & Reference & $1.96(1.44-2.65)$ & $3.02(2.17-4.22)$ \\
\hline MUHO & Reference & $2.29(1.46-3.59)$ & $5.38(3.45-9.39)$ \\
\hline \multicolumn{4}{|l|}{ MUHL } \\
\hline Model 1 & Reference & $1.25(0.29-5.36)$ & $2.33(0.45-11.93)$ \\
\hline Model2 & Reference & $1.16(0.26-5.14)$ & $2.04(0.37-11.12)$ \\
\hline Model3 & Reference & $0.73(0.13-4.02)$ & $1.69(0.30-9.41)$ \\
\hline \multicolumn{4}{|l|}{$\mathrm{MHO}$} \\
\hline Model1 & Reference & $2.57(1.85-3.58)$ & $5.18(3.49-7.70)$ \\
\hline Model2 & Reference & $2.62(1.88-3.66)$ & $5.45(3.64-8.17)$ \\
\hline Model 3 & Reference & $2.63(1.88-3.68)$ & $5.61(3.73-8.43)$ \\
\hline \multicolumn{4}{|l|}{ MUHO } \\
\hline Model 1 & Reference & $3.07(1.91-4.94)$ & $9.72(5.74-16.45)$ \\
\hline Model2 & Reference & $3.17(1.96-5.11)$ & $10.73(6.29-18.031)$ \\
\hline Model3 & Reference & $3.22(1.99-5.22)$ & 11.16(6.51-19.11) \\
\hline
\end{tabular}

The highest odds for MUHO were observed in model 3 (i.e., adjusting for age, gender, marital status, education level, smoking, and alcohol consumption), which was $3.53(95 \% \mathrm{Cl} 2.11-5.90)$ in the second SUA tertile and $14.29(95 \% \mathrm{Cl} 8.15-25.08)$ in the third SUA tertile. In contrast, no similar trend was observed for the association between MUHL and SUA tertile.

\section{Discussion}


We assessed the relationship between SUA and the cardiometabolic phenotype in the current study, and our findings show increasing mean values of metabolic syndrome factors, including LDL and cholesterol, in a dose-response manner corresponding to the SUA tertiles. These results are similar to the findings of the studies carried out previously in various countries [16-18]. According to our findings, the prevalence rates of $\mathrm{MHO}$ and $\mathrm{MUHL}$ were higher in the second and third tertiles compared to the first tertile, which had the lowest SUA. As far as we know, no studies have been published focusing on the relationship between SUA and the cardiometabolic phenotype. Therefore, we contrasted our results with previous studies that evaluated the connection between SUA and MetS. An increase in MetS due to the elevation of serum uric acid level has been reported [19]. The results of the current study are in line with the findings of Ishizaka et al., who evaluated the relationship between SUA and MetS among participants with $\mathrm{BMl} \geq 25 \mathrm{~kg} / \mathrm{m}^{2}$. They noticed that in the highest uric acid value quartile, the odds of MetS elevated by 2.27 (95\% Cl: 1.90-2.72) after adjusting for the confounding factors [20]. However, in our study, after adjusting for the confounding factors, the risk of $\mathrm{MUHO}$ in the highest tertile was more than that of the study by Ishizaka et al. (OR 11.16 vs 2.27 ). This variation is potentially due to the differences in defining MetS, i.e., they considered BMI $>25 \mathrm{~kg} / \mathrm{m}^{2}$ as a MetS marker, while we determined MetS according to ATP III, and then categorized the participants based on BMI (BMI<25 or $\left.\geq 25 \mathrm{~kg} / \mathrm{m}^{2}\right)$.

Hemostasis and the relationship between SUA homeostasis and MetS are highly complex [21]. It's still debatable whether an elevated SUA level is a risk factor or just a biomarker in the progress and improvement of MetS [22]. Some researchers have stated that hyperuricemia can be an exclusive component of MetS $[7,23]$, while other studies have proposed to consider hyperuricemia as a supplementary component of MetS [24, 25]. Elevated SUA levels will cause outcomes such as hypertension [26], hypertriglyceridemia, and hypercholesterolemia [27]. The suggested procedures for the connection between SUA and MetS include the following: firstly, hyperuricemia has been proved to lead to endothelial dysfunction in human and animal bodies [28, 29]. Secondly, SUA has been shown to prevent NO production [30], which is a significant factor in the functioning of insulin [31]. The defect of endothelial-formed NO is supposed to decrease blood flow to the cells, which stops the normal functioning of insulin and causes hyperinsulinemia [21]. Therefore, hyperuricemia may play a potential role in causing and increasing insulin resistance. Similarly, insulin resistance is recognized to play an essential role in the pathogenesis of MetS [32]. Thirdly, another role of uric acid involves inducing oxidative stress, which causes inflammation in adipocytes [33,34] and hepatocytes [35]. However, the complicated correlation between uric acid and oxidative stress is noteworthy because it can be paradoxical [36]. Although uric acid is an antioxidant that disables superoxide anion, peroxynitrite, and hydroxyl radicals [37, 38], there is some evidence showing that under ischemic stress or high SUA, uric acid functions as a pro-oxidant.

Furthermore, we noticed that the prevalence of males in the third tertile (i.e., the highest SUA level) was significantly higher than the other tertiles. In line with our results, this phenomenon has been mentioned in previous studies $[39,40]$. It seems that the lower tubular urate post-secretory reabsorption and the higher renal clearance of urate in women can be related to this observation. 
Moreover, according to our findings, the average serum levels of liver enzymes were elevated in $\mathrm{MHL}$, $\mathrm{MHO}$, and MUHO individuals in a dose-response manner corresponding to SUA tertiles. Interestingly, the mean serum liver enzymes elevated with increasing SUA levels in MHL individuals. This may suggest an association between liver enzymes and SUA that is independent of BMI. At the same time, it may be an indicator that the MHL subjects in the third tertile are at risk of shifting to MUHL. Nevertheless, we did not observe the same trend in the MUHL group, which we thought might be because of the limited sample size of this group. These findings are in line with the findings of prior studies [41, 42]. For instance, Shih et al. state that individuals with hyperuricemia are more likely to have heightened liver enzymes (AST or ALT), even after adjustment [41]. As shown in several studies, NAFLD is closely linked with obesity, dyslipidemia, diabetes mellitus, MetS, and cardiovascular disorders [43,44]. Therefore, NAFLD is believed to be a hepatic outcome of metabolic diseases $[45,46]$. It turns out that the SUA level increases in most NAFLD patients [47], indicating that it can be an independent predisposing factor for NAFLD [48, 49]. Additionally, hyperuricemia, even in the reference range, was a component of MetS [50].

The main limitation of the current study involves the fact that due to its cross-sectional design, causal inferences in the relationship between serum SUA and cardiometabolic phenotype could not be evaluated. However, the main strength of this study is that it is the first to evaluate the relationship between SUA and cardiometabolic phenotype in healthcare workers. Serum SUA is easily accessible in regular clinical practice, and it is measured using standardized techniques. It would be useful to distinguish the transition from $\mathrm{MHO}$ to $\mathrm{MUHO}$. Therefore, it may lead to earlier and more precise identification of $\mathrm{MHO}$ subjects at risk of transition to MUHL, which can facilitate the administration of better preventive strategies. Another strong point of this work lies in using data from a cohort study and a large sample size.

\section{Conclusions}

Our findings indicate that increases in the prevalence of $\mathrm{MHO}$ and $\mathrm{MUHO}$ are related to elevated SUA levels. Furthermore, the average MetS components and lipid profile increased with the elevation in SUA levels. Additionally, there is a positive dose-response manner associated with the mean serum liver enzymes in MHL, MHO, and MUHO groups. Accordingly, we proposed the use of the easily-measured SUA level as a marker for the early diagnosis of at-risk MUHL and MHO individuals in order to provide proper intervention. However, the detailed mechanisms that cause SUA to lead to this disorder are still at an early stage of investigation, and they need further explanation. Consequently, further prospective works are needed to identify the effects of SUA on the progression of MetS in various body-size subgroups.

\section{Declarations}

\section{Ethics approval and consent to participate}

This study was approved by the ethic committee of Tabriz University of medical sciences (IR.TBZMED.REC.1396.1263). 


\section{Availability of data and materials}

The data that support the findings of this study are available from [Vice Chancellor for Research] but restrictions apply to the availability of these data, which were used under license for the current study, and so are not publicly available. Data are however available from the authors upon reasonable request and with permission of [Vice Chancellor for Research]

\section{Competing interests}

The authors declare that they have no competing interests

\section{Funding}

This study was supported by the liver and gastrointestinal diseases research center, Tabriz University of Medical Sciences. The funder had no role on the study design, data analysis, interpreting and writing the manuscript in this study.

\section{Authors' contributions}

-The conception or design of the work: MHS,ARO

-The acquisition, analysis: NG,EF

OR interpretation of data: EF

Drafted the work or substantively revised: EF,NF,SZ

All authors have read and approved the manuscript

\section{Consent for publication}

Not Applicable

\section{Acknowledgments}

The authors also are deeply indebted to all subjects who participated in this study. In addition, we would like to thank the Persian cohort study staff for their technical support.

\section{References}

1. Biradar MI, Chiang K-M, Yang H-C, Huang Y-T, Pan W-H. The causal role of elevated uric acid and waist circumference on the risk of metabolic syndrome components. Inter J Obesity.2020; 44(4):865874.

2. Misra A, Khurana L. Obesity and the metabolic syndrome in developing countries. The $\mathrm{J}$ Clin Endocrinol Metabolism. 2008; 93(11_supplement_1):s9-s30. 
3. Hosseini-Esfahani F, Bahadoran Z, Moslehi N, Asghari G, Yuzbashian E, Hosseinpour-Niazi S, etal. Metabolic syndrome: Findings from 20 years of the Tehran Lipid and Glucose Study. Inter J Endocrinol Metabolism. 2018; 16(4 Suppl).

4. Zapolski T, Waciński P, Kondracki B, Rychta E, Buraczyńska MJ, Wysokiński A. Uric acid as a link between renal dysfunction and both pro-inflammatory and prothrombotic state in patients with metabolic syndrome and coronary artery disease. Kardiologia Polska (Polish Heart Journal). 2011; 69(4):319-326.

5. Sutherland JP, McKinley B, Eckel RH. The metabolic syndrome and inflammation. Metabolic Syndrome Related Disorder. 2004;2(2):82-104.

6. Wang L, Zhang T, Liu Y, Tang F, Xue F. Association of Serum Uric Acid with Metabolic Syndrome and Its Components: A Mendelian Randomization Analysis. BioMed Res Inter.2020; 2020.

7. Yoo TW, Sung KC, Shin HS, Kim BJ, Kim BS, Kang JH, etal.Relationship between serum uric acid concentration and insulin resistance and metabolic syndrome. Circulation J. 2005; 69(8):928-933.

8. Yu TY, Jee JH, Bae JC, Jin S-M, Baek J-H, Lee M-K, etal. Serum uric acid: A strong and independent predictor of metabolic syndrome after adjusting for body composition. Metabolism. 2016; 65(4):432440.

9. Ishizaka N, Ishizaka Y, Toda A, Tani M, Koike K, Yamakado M, etal. Changes in waist circumference and body mass index in relation to changes in serum uric acid in Japanese individuals. J Rheumatol. 2010; 37(2):410-416.

10. Ali N, Perveen R, Rahman S, Mahmood S, Rahman S, Islam S, etal. Prevalence of hyperuricemia and the relationship between serum uric acid and obesity: A study on Bangladeshi adults. PLoS One. 2018; 13(11):e0206850.

11. Velho S, Paccaud F, Waeber G, Vollenweider P, Marques-Vidal P. Metabolically healthy obesity: different prevalences using different criteria. Eur J Clin Nutr. 2010; 64(10):1043-1051.

12. Wildman RP, Muntner P, Reynolds K, McGinn AP, Rajpathak S, Wylie-Rosett J, etal.The obese without cardiometabolic risk factor clustering and the normal weight with cardiometabolic risk factor clustering: prevalence and correlates of 2 phenotypes among the US population (NHANES 19992004). Arch Inter Med. 2008; 168(15):1617-1624.

13. Poustchi H, Eghtesad S, Kamangar F, Etemadi A, Keshtkar A-A, Hekmatdoost A, etal. Prospective epidemiological research studies in Iran (the PERSIAN Cohort Study): rationale, objectives, and design. Am J Epidemiol. 2018; 187(4):647-655.

14. Farhang S, Faramarzi E, Amini Sani N, Poustchi H, Ostadrahimi A, Alizadeh BZ, etal.Cohort Profile: The AZAR cohort, a health-oriented research model in areas of major environmental change in Central Asia. Inter J Epidemiol. 2019; 48(2):382-382h.

15. Alberti K, Eckel RH, Grundy SM, Zimmet PZ, Cleeman JI, Donato KA, etal. Harmonizing the metabolic syndrome: a joint interim statement of the international diabetes federation task force on epidemiology and prevention; national heart, lung, and blood institute; American heart association; 
world heart federation; international atherosclerosis society; and international association for the study of obesity. Circulation. 2009; 120(16):1640-1645.

16. Baygi F, Herttua K, Sheidaei A, Ahmadvand A, Jensen OC. Association of Serum Uric Acid with cardiometabolic risk factors and metabolic syndrome in seafarers working on tankers. BMC Public Health .2020; 20:1-6.

17. Nurshad A, Rakib M, Mahmudul H, Zitu B, Mou AD, Momtahena HJ, etal. Association between serum uric acid and metabolic syndrome: a cross-sectional study in Bangladeshi adults. Scient Report . 2020; 10(1).

18. Jeong J, Suh YJ. Association between serum uric acid and metabolic syndrome in Koreans. J Korean Med Scien. 2019; 34(48).

19. Choi HK, Ford ES. Prevalence of the metabolic syndrome in individuals with hyperuricemia. Am J Med. 2007; 120(5):442-447.

20. Ishizaka N, Ishizaka Y, Toda E-I, Nagai R, Yamakado M.Association between serum uric acid, metabolic syndrome, and carotid atherosclerosis in Japanese individuals. Arterioscler Thromb Vascular Biol. 2005;25(5):1038-1044.

21. Codoñer-Franch P, Tavárez-Alonso S, Murria-Estal R, Megías-Vericat J, Tortajada-Girbés M, AlonsoIglesias E. Nitric oxide production is increased in severely obese children and related to markers of oxidative stress and inflammation. Atheroscler. 2011;215(2):475-480.

22. Wang H-J, Shi L-Z, Liu C-F, Liu S-M, Shi S-T. Association between uric acid and metabolic syndrome in elderly women. Open Med. 2018;13(1):172-177.

23. Tsouli SG, Liberopoulos EN, Mikhailidis DP, Athyros VG, Elisaf MS. Elevated serum uric acid levels in metabolic syndrome: an active component or an innocent bystander? Metabolism. 2006;55(10):1293-1301.

24. Liou T-L, Lin M-W, Hsiao L-C, Tsai T-T, Chan W-L, Ho L-T, Hwu C-M.Is hyperuricemia another facet of the metabolic syndrome? J Chin MedAssociat. 2006;69(3):104-109.

25. Sheu WH, Tseng Y-H.Uric acid: an additional component of metabolic syndrome? J Chin Med Associat. 2006;69(3):99-100.

26. Han G, Gonzalez S, DeVries D. Combined effect of hyperuricemia and overweight/obesity on the prevalence of hypertension among US adults: result from the National Health and Nutrition Examination Survey. J Human Hypertension. 2014;28(10):579-586.

27. Loeffler LF, Navas-Acien A, Brady TM, Miller III ER, Fadrowski JJ. Uric acid level and elevated blood pressure in US adolescents National Health and Nutrition Examination Survey, 1999-2006. Hypertension. 2012; 59(4):811-817.

28. Khosla UM, Zharikov S, Finch JL, Nakagawa T, Roncal C, Mu W, etal. Hyperuricemia induces endothelial dysfunction. Kidney Inter. 2005; 67(5):1739-1742.

29. Mercuro G, Vitale C, Cerquetani E, Zoncu S, Deidda M, Fini M, etal. Effect of hyperuricemia upon endothelial function in patients at increased cardiovascular risk. The Amer J Cardiol.2004; 94(7):932935. 
30. Kang D-H, Park S-K, Lee I-K, Johnson RJ. Uric acid-induced C-reactive protein expression: implication on cell proliferation and nitric oxide production of human vascular cells. J Am Society Nephrol. 2005; 16(12):3553-3562.

31. Roy D, Perreault M, Marette A. Insulin stimulation of glucose uptake in skeletal muscles and adipose tissues in vivo is NO dependent. Am J Physiol Endocrinol Metab. 1998; 274(4):E692-E699.

32. Lanaspa MA, Sanchez-Lozada LG, Choi Y-J, Cicerchi C, Kanbay M, Roncal-Jimenez CA, etal. Uric Acid induces hepatic steatosis by generation of mitochondrial oxidative stress potential role in fructosedependent and-independent fatty liver. J Biol Chem. 2012; 287(48):40732-40744.

33. Baldwin W, McRae S, Marek G, Wymer D, Pannu V, Baylis C, etal. Hyperuricemia as a mediator of the proinflammatory endocrine imbalance in the adipose tissue in a murine model of the metabolic syndrome. Diabetes. 2011; 60(4):1258-1269.

34. Sautin YY, Nakagawa T, Zharikov S, Johnson RJ. Adverse effects of the classic antioxidant uric acid in adipocytes: NADPH oxidase-mediated oxidative/nitrosative stress. Am J PhysiolCell Physiol. 2007; 293: C584-C596.

35. Choi Y-J, Shin H-S, Choi HS, Park J-W, Jo I, Oh E-S, etal. Uric acid induces fat accumulation via generation of endoplasmic reticulum stress and SREBP-1c activation in hepatocytes. Lab Invest. 2014; 94(10):1114-1125.

36. Lippi G, Montagnana M, Franchini M, Favaloro EJ, Targher G. The paradoxical relationship between serum uric acid and cardiovascular disease. Clin Chim Acta. 2008; 392(1-2):1-7.

37. Ames BN, Cathcart R, Schwiers E, Hochstein P. Uric acid provides an antioxidant defense in humans against oxidant-and radical-caused aging and cancer: a hypothesis. Proceed Nation Acad Scien. 1981; 78(11):6858-6862.

38. Robinson KM, Morré JT, Beckman JS. Triuret: a novel product of peroxynitrite-mediated oxidation of urate. Arch Biochem Biophy. 2004; 423(1):213-217.

39. Sui X, Church TS, Meriwether RA, Lobelo F, Blair SN. Uric acid and the development of metabolic syndrome in women and men. Metabolism. 2008;57(6):845-852.

40. Conen D, Wietlisbach V, Bovet P, Shamlaye C, Riesen W, Paccaud F, etal. Prevalence of hyperuricemia and relation of serum uric acid with cardiovascular risk factors in a developing country. BMC Public Health. 2004; 4(1):9.

41. Shih M-H, Lazo M, Liu S-H, Bonekamp S, Hernaez R, Clark JM. Association between serum uric acid and nonalcoholic fatty liver disease in the US population. J Formosan Med Associat. 2015; 114(4):314-320.

42. Zelber-Sagi S, Ben-Assuli O, Rabinowich L, Goldstein A, Magid A, Shalev V, Shibolet O, Chodick G. The association between the serum levels of uric acid and alanine aminotransferase in a populationbased cohort. Liver Inter. 2015; 35(11):2408-2415.

43. Goessling W, Massaro JM, Vasan RS, D'Agostino Sr RB, Ellison RC, Fox CS. Aminotransferase levels and 20-year risk of metabolic syndrome, diabetes, and cardiovascular disease. Gastroenterol. 2008; 135(6):1935-1944. e1931. 
44. Choi SS, Diehl AM. Hepatic triglyceride synthesis and nonalcoholic fatty liver disease. Curr Opinion lipidol. 2008; 19(3):295-300.

45. Angelico F, Del Ben M, Conti R, Francioso S, Feole K, Maccioni D, etal. Non-alcoholic fatty liver syndrome: a hepatic consequence of common metabolic diseases. J Gastroenterol Hepatol. 2003; 18(5):588-594.

46. Cortez-Pinto H, Camilo M, Baptista A, De Oliveira A, De Moura M. Non-alcoholic fatty liver: another feature of the metabolicsyndrome? Clin Nutr.1999; 18(6):353-358.

47. Marchesini G, Brizi M, Bianchi G, Tomassetti S, Bugianesi E, Lenzi M, etal.Nonalcoholic fatty liver disease: a feature of the metabolic syndrome. Diabete. 2001; 50(8):1844-1850.

48. Sirota JC, McFann K, Targher G, Johnson RJ, Chonchol M, Jalal DI.Elevated serum uric acid levels are associated with non-alcoholic fatty liver disease independently of metabolic syndrome features in the United States: Liver ultrasound data from the National Health and Nutrition Examination Survey. Metabolism. 2013; 62(3):392-399.

49. Lee Y-J, Lee H-R, Lee J-H, Shin Y-H, Shim J-Y. Association between serum uric acid and non-alcoholic fatty liver disease in Korean adults. Clin Chem Lab Med. 2010; 48(2):175-180.

50. Nejatinamini S, Ataie-Jafari A, Qorbani M, Nikoohemat S, Kelishadi R, Asayesh H, Hosseini S. Association between serum uric acid level and metabolic syndrome components. J Diabetes Metabolic Disord. 2015; 14(1):70. 